\title{
HYPERPLANE SECTIONS OF LEGENDRIAN SUBVARIETIES
}

\author{
JAROSŁAW BUCZYŃSKI
}

\begin{abstract}
We prove that a general hyperplane section of a smooth Legendrian subvariety in a projective space admits Legendrian embedding into another projective space. This gives numerous new examples of smooth Legendrian subvarieties, some of which have positive Kodaira dimension.
\end{abstract}

\section{Introduction and statement of results}

The main object of our studies are Legendrian subvarieties in projective space.

Definition. Let $\omega$ be a symplectic form on $V=\mathbb{C}^{2 n+2}$. A subvariety $X \subset \mathbb{P}(V)$ is Legendrian, if for each smooth point of its affine cone $\hat{X}$ the tangent space to $\hat{X} \subset V$ at this point is Lagrangian, i.e., maximal isotropic with respect to $\omega$.

Prior to this paper, one of the problems regarding smooth Legendrian subvarieties of $\mathbb{P}^{2 n+1}$ has been the lack of examples. It has been hoped, that in higher dimensions there is only a few of examples, mainly some homogeneous varieties, so called subadjoint varieties (see [6], [2], [8]). Strong restrictions on topology of smooth Legendrian varieties have been found and studied by Landsberg and Manivel.

In this article we prove:

Theorem 1.1. Let $X \subset \mathbb{P}(V)$ be an irreducible Legendrian subvariety, which is smooth or has only isolated singularities. Then a general hyperplane section of $X$ admits a Legendrian embedding into a projective space of an appropriate dimension via a specific subsystem of the linear system $\mathcal{O}(1)$.

More generally, assume $X \subset \mathbb{P}(V)$ is an irreducible Legendrian subvariety with singular locus of dimension $k$ and $H \subset \mathbb{P}(V)$ is a general hyperplane. Then there exists a variety $\widetilde{X}_{H}$ whose singular locus has dimension at most $k-1$ and which has an open subset isomorphic to the smooth locus of $X \cap H$ such that $\widetilde{X}_{H}$ admits a Legendrian embedding.

The specific linear system and construction of $\widetilde{X}_{H}$ is described in section 2.1 and there we prove that the resulting variety is Legendrian. The proof that for a general section the result has the required smoothness property is presented in section 2.2.

This simple observation has quite strong consequences. Many researchers, including Landsberg, Manivel, Wiśniewski, Hwang and the author of this article, believed that the structure of smooth Legendrian subvarieties in projective space had to be somehow rigid at least in higher dimensions. So far the only non-rational examples were known in dimensions 1 (see $[1$, thm G]) and 2 (see $[6, \S 4]$ ) and these were also

Received by the editors July 3, 2007. 
the only known to come in families. Now applying our theorem to the subadjoint varieties we get many more examples with various properties:

Example 1.2. The following smooth varieties and families of smooth varieties admit Legendrian embedding:

(a) a family of $K 3$ surfaces of genus 9;

(b) three different types of surfaces of general type;

(c) some Calabi-Yau 3-folds, some Calabi-Yau 5-folds and some Calabi-Yau 9folds;

(d) some varieties of general type in every of dimensions 3, 4 (two families for every dimension), 5,6,7 and 8 (one family per dimension).

(e) some Fano varieties, like the blow up of a quadric $Q^{n}$ in a codimension 2 hyperplane section $Q^{n-2}$, a family of Del Pezzo surfaces of degree 4 and others;

(f) infinitely many non-isomorphic, non-homogeneous Legedrian varieties in every dimension arising as a codimension $k$ linear section of $\mathbb{P}^{1} \times Q^{n+k}$.

Example (a) agrees with the prediction of [6, §2.3]. Examples (b) and (d) give a partial answer to the question of a possible Kodaira dimension of a Legendrian variety (also see $[6, \S 2.3]$ ). Example (f) is a contradiction to the naive expectation that Legendrian variety in a sufficiently high dimension must be homogeneous.

We also note that previous examples of the author also arise in this way. Example (e) for $n=2$ is described in [4, ex. 3.4]. Hyperplane sections of $G r(3,6), G r_{L}(3,6), \mathbb{S}_{6}$ are studied in more details in [3]. Also non-homogeneous examples of other authors, Bryant [1], Landsberg and Manivel [6] can be reconstructed by theorem 1.1 from some varieties with only isolated singularities (see section 3 ).

All the varieties arising from theorem 1.1 and our construction in subsection 2.1 are embedded by a non-complete linear system. Therefore a natural question arises: what are the smooth Legendrian varieties whose Legendrian embedding is linearly normal. Another question is whether the construction can be inverted. So for a given Legendrian, but not linearly normal embedding of some variety $\widetilde{X}$, can we find a bigger Legendrian variety $X$, such that $\widetilde{X}$ is a projection of a hyperplane section of $X$ ?

Building upon ideas of Bryant, Landsberg and Manivel we suggest a construction that provides some (but far from perfect) answer for the second question in section 3. In particular we represent the example of Landsberg and Manivel as a hyperplane section of a 3-fold with only isolated singularities and the examples of Bryant as hyperplane sections of surfaces with at most isolated singularities.

Legendrian varieties arise as varieties of tangent directions to minimal rational curves on contact manifolds (see [2, §2] and references therein for a brief review on the subject). Having many examples of smooth Legendrian varieties (as well as families of such), can we construct a new example of a contact manifold, whose variety of tangent directions to minimal rational curves is one of the Legendrian varieties (or is in the given family)? It is unlikely that the answer is positive, but if not, then what are the obstructions, i.e., what conditions should we request for the Legendrian variety to make the reconstruction of contact manifold possible? 


\section{Hyperplane section}

2.1. Construction. The construction is as follows. Let $H \in \mathbb{P}\left(V^{*}\right)$ be a hyperplane in $V$. By

$$
h:=H^{\perp_{\omega}} \subset V
$$

we denote the $\omega$-perpendicular to $H$ subspace of $V$, which in this case is a line contained in $H$. We think of $h$ both as a point in the projective space $\mathbb{P}(V)$ and a line in $V$. We define

$$
\pi: \mathbb{P}(H) \backslash\{h\} \longrightarrow \mathbb{P}(H / h)
$$

to be the projection map and for a given Legendrian subvariety $X \subset \mathbb{P}(V)$ we let $\widetilde{X}_{H}:=\pi(X \cap H)$.

We have a natural symplectic structure $\omega^{\prime}$ on $H / h$ determined by $\omega$. Also $\widetilde{X}_{H}$ is always isotropic (i.e., the tangent bundle to $\widetilde{X}_{H}$ is contained in the contact distribution) as the projection map $H \longrightarrow H / h$ preserves the two 2 -forms $\left.\omega\right|_{H}, \omega^{\prime}$ and moreover, every map of algebraic varieties is generically a submersion onto the image.

Also unless $X$ is a cone with vertex $h$ (so the choice of $H$ is very special), the dimension $\operatorname{dim} \widetilde{X}_{H}=\operatorname{dim}(X \cap H)=\operatorname{dim} X-1$ so that $X$ is Legendrian.

Note that so far we have not used any smoothness condition on $X$.

2.2. Proof of smoothness. Hence to prove theorem 1.1 it is enough to prove that for a general $H \in \mathbb{P}\left(V^{*}\right)$, the map $\pi$ gives an isomorphism of the smooth locus of $X \cap H$ onto its image, an open subset in $\widetilde{X}_{H}$.

For a variety $Y \subset \mathbb{P}^{m}$ we denote by $\sigma(Y) \subset \mathbb{P}^{m}$ its secant variety, i.e., closure of the union of all projective lines through $y_{1}$ and $y_{2}$, where $\left(y_{1}, y_{2}\right)$ vary through pairs of different points of $Y$.

Lemma 2.1. Let $Y \subset \mathbb{P}^{m}$, choose such a point $y \in \mathbb{P}^{m}$ that $y \notin \sigma(Y)$ and let $\pi: \mathbb{P}^{m} \backslash\{y\} \longrightarrow \mathbb{P}^{m-1}$ be the projection map.

(a) If $Y$ is smooth then $\pi$ gives an isomorphism of $Y$ and $\pi(Y)$.

(b) In general, $\pi$ is 1 to 1 and $\pi$ is an isomorphism of the smooth part of $Y$ onto its image. In particular the dimension of singular locus of $Y$ is greater or equal to the dimension of singular locus of $\pi(Y)$.

Proof. See [5, prop. IV.3.4 and exercise IV.3.11(a)]. We only note, that if $Y$ is smooth, then the secant variety $\sigma(Y)$ contains all the embedded tangent spaces of $Y$. They arise when $y_{2}$ approaches $y_{1}$.

Now we can prove theorem 1.1:

Proof. By the lemma and the construction in subsection 2.1 it is enough to prove that there exists $h \in \mathbb{P}(V)$ s.t. $h \notin \sigma\left(X \cap h^{\perp_{\omega}}\right)$.

Given two different points $x_{1}$ and $x_{2}$ in a projective space we denote by $\left\langle x_{1}, x_{2}\right\rangle$ the projective line through $x_{1}$ and $x_{2}$. Let:

$$
\tilde{\sigma}(X) \subset X \times X \times \mathbb{P}(V), \tilde{\sigma}(X):=\overline{\left\{\left(x_{1}, x_{2}, p\right) \mid p \in\left\langle x_{1}, x_{2}\right\rangle\right\}}
$$

so that $\tilde{\sigma}(X)$ is the incidence variety for the secant variety of $X$. Obviously, $\operatorname{dim}(\tilde{\sigma}(X))=2 \operatorname{dim} X+1=\operatorname{dim}(\mathbb{P}(V))$. Also we let:

$$
\kappa(X) \subset \tilde{\sigma}(X), \kappa(X):=\overline{\left\{\left(x_{1}, x_{2}, h\right) \mid h \in\left\langle x_{1}, x_{2}\right\rangle \text { and } x_{1}, x_{2} \in h^{\perp \omega}\right\}} .
$$


so that the image of the projection of $\kappa(X)$ onto the last coordinate is the locus of 'bad' points. More precisely, for a point $h \in \mathbb{P}(V)$ there exist $\left(x_{1}, x_{2}\right)$ such that $\left(x_{1}, x_{2}, h\right) \in \kappa(X)$ if and only if $h \in \sigma\left(X \cap h^{\perp \omega}\right)$.

We claim that the image of $\kappa(X)$ under the projection is not whole the $\mathbb{P}(V)$. To see that note, that the condition defining $\kappa(X)$, i.e., $h \in\left\langle x_{1}, x_{2}\right\rangle, x_{1}, x_{2} \in h^{\perp_{\omega}}$ is equivalent to $h \in\left\langle x_{1}, x_{2}\right\rangle$ and $\left\langle x_{1}, x_{2}\right\rangle$ is an isotropic subspace of $V$. Now either $X$ is a linear subspace and then both the claim and the theorem are obvious or there exist two points $x_{1}, x_{2} \in X$ such that $\omega\left(\hat{x_{1}}, \hat{x_{2}}\right) \neq 0$ where by $\hat{x_{i}}$ we mean some non-zero point in the line $x_{i} \subset V$. Therefore $\kappa(X)$ is strictly contained in $\tilde{\sigma}(X)$ and

$$
\operatorname{dim}(\kappa(X))<\operatorname{dim}(\tilde{\sigma}(X))=\operatorname{dim} \mathbb{P}(V),
$$

so the image of $\kappa(X)$ under the projection cannot be equal to $\mathbb{P}(V)^{1}$.

Corollary 2.2. Let $X \subset \mathbb{P}(V)$ be an irreducible Legendrian subvariety whose singular locus has dimension at most $k-1$. If $H \subset \mathbb{P}(V)$ is a general coisotropic linear subspace of codimension $k$, then $\widetilde{X}_{H}:=X \cap H$ is smooth and admits a Legendrian embedding via an appropriate subsystem of linear system $\mathcal{O}_{\widetilde{X}}(1)$.

We sketch some proofs for examples 1.2:

Proof. $K 3$ surfaces of (a) arise as codimension 4 linear sections of Lagrangian Grassmannian $G r_{L}(3,6)$. Since the canonical divisor $K_{G r_{L}(3,6)}=\mathcal{O}_{G r_{L}(3,6)}(-4)$ (in other words $\operatorname{Gr}_{L}(3,6)$ is Fano of index 4), by the adjunction formula, the canonical divisor of the section is indeed trivial. On the other hand, by [6, prop. 9] it must have genus 9. Although we take quite special (coisotropic) sections, they fall into the 19 dimensional family of Mukai genus $9 \mathrm{~K} 3$-surfaces [7] and they form a 13 dimensional subfamily.

The other families of surfaces as in (b) arise as sections of the other exceptional subadjoint varieties: $\operatorname{Gr}(3,6), \mathbb{S}_{6}$ and $E_{7}$. Since they are all Fano of index 6,10 and 18 respectively and their dimensions are 9, 15 and 27 hence taking successive linear sections we get to Calabi-Yau manifolds as stated in (c) and further the canonical divisor is very ample, so we have examples of general type as stated in (b) and (d).

The Fano varieties arise as intermediate steps, before coming down to the level of Calabi-Yau manifolds. Also $\mathbb{P}^{1} \times Q^{n}$ is a subadjoint variety and its hyperplane section is the blow up of a quadric $Q^{n}$ in a codimension 2 hyperplane section. The Del Pezzo surfaces are the hyperplane sections of the blow up of $Q^{3}$ in a conic curve.

\section{Extending Legendrian varieties}

Our motivation is the example of Landsberg and Manivel [6, §4], a Legendrian embedding of a Kummer K3 surface blown up in 12 point. It can be seen, that this embedding is given by a codimension 1 linear system. We want to find a Legendrian 3 -fold in $\mathbb{P}^{7}$ whose hyperplane section is this example. Unfortunately, we are not

\footnotetext{
${ }^{1}$ The inequality on the dimenisons, although simple, is essential for the proof. An analogous construction for Lagrangian subvarieties in symplectic manifolds is known as symplecic reduction, but does not produce smooth Lagrangian subvarieties.
} 
able to find a smooth 3-fold with these properties, but we get one with only isolated singularities.

We recall the setup for the construction of the example. Let $W$ be a vector space of dimension $n+1$. Let $Z$ be any subvariety in $\mathbb{P}^{n}=\mathbb{P}(W)$ and let $Z^{*} \subset \check{\mathbb{P}}^{n}=\mathbb{P}\left(W^{*}\right)$ be its dual variety in the dual projective space. Also let $Z^{\sharp} \subset \mathbb{P}\left(T^{*} \mathbb{P}^{n}\right) \subset \mathbb{P}^{n} \times \check{\mathbb{P}}^{n}$ be the conormal variety, i.e., the closure of the union of projectivised conormal spaces over smooth points of $Z$. Landsberg and Manivel study in details an explicit birational $\operatorname{map} \varphi:=\varphi_{H_{0}, p_{0}}: \mathbb{P}\left(T^{*} \mathbb{P}^{n}\right) \rightarrow \mathbb{P}^{2 n-1}$ which depends on a hyperplane $H_{0}$ in $\mathbb{P}^{n}$ and on a point $p_{0} \in H_{0}$. After Bryant [1] they observe that $\varphi(Z)$ (if only makes sense) is always a Legendrian subvariety, but usually singular. Next they study conditions under which $\varphi(Z)$ is smooth. In particular they prove that the conditions are satisfied when $Z$ is a Kummer quartic surface in $\mathbb{P}^{3}$ in general position with respect to $p_{0}$ and $H_{0}$ and this gives rise to their example.

We want to modify the above construction just a little bit to obtain our 3-fold. Instead of considering $Z^{\sharp}$ as a subvariety in

$$
\mathbb{P}(W) \times \mathbb{P}\left(W^{*}\right)=(W \backslash\{0\}) \times\left(W^{*} \backslash\{0\}\right) / \mathbb{C}^{*} \times \mathbb{C}^{*},
$$

we consider a subvariety $X$ in

$$
\mathbb{P}^{2 n+1}=\mathbb{P}\left(W \oplus W^{*}\right)=\left(W \times W^{*}\right) \backslash\{0\} / \mathbb{C}^{*}
$$

such that the underlying affine cone of $X$ in $W \times W^{*}$ is the same as the underlying affine pencil of $Z^{\sharp}$. In other words, we take $X$ to be the closure of preimage of $Z^{\sharp}$ under the natural projection map:

$$
p: \mathbb{P}\left(W \oplus W^{*}\right) \rightarrow \mathbb{P}(W) \times \mathbb{P}\left(W^{*}\right) .
$$

Theorem 3.1. Let $X \subset \mathbb{P}\left(W \oplus W^{*}\right)$ be a subvariety constructed as above from any irreducible subvariety $Z \subset \mathbb{P}(W)$. Then:

(i) $X$ is a Legendrian subvariety contained in the quadric $p^{-1}\left(\mathbb{P}\left(T^{*} \mathbb{P}(W)\right)\right)$.

(ii) Choose $H$ to be a hyperplane section of $\mathbb{P}\left(W \oplus W^{*}\right)$ which does not contain $\mathbb{P}(W)$ nor $\mathbb{P}\left(W^{*}\right)$. Set $H_{0}:=\mathbb{P}(W) \cap H$ and $p_{0}$ to be the point in $\mathbb{P}(W)$ dual to $\mathbb{P}\left(W^{*}\right) \cap H$ and assume $H$ is chosen in such a way that $p_{0} \in H_{0}$. So we have two Legendrian subvarieties in $\mathbb{P}^{2 n-1}$ : one is the closure of $\varphi_{H_{0}, p_{0}}\left(Z^{\sharp}\right)$ as in the construction of $[6, \S 4]$ and the other (as in subsection 2.1) is the image under projection from $H^{\perp_{\omega}}$ of $X \cap H$. Then the two constructions agree, i.e., $\varphi_{H_{0}, p_{0}}\left(Z^{\sharp}\right)$ is a component of the image of $X \cap H$.

(iii) $X$ is singular at the following points: on $X \cap \mathbb{P}(W)$ at singular points of $Z \subset$ $\mathbb{P}(W)$, on $X \cap \mathbb{P}\left(W^{*}\right)$ at the singular points of $Z^{*}$ and outside $\mathbb{P}(W) \cup \mathbb{P}\left(W^{*}\right)$ at the preimage under $p$ of the singular points of the conormal variety $Z^{\sharp}$.

Proof. Part (i) is an easy verification - simply the affine tangent space to $X$ at a general point $[w, \alpha] \in X \subset \mathbb{P}\left(W \oplus W^{*}\right)$ is the sum $T_{[w]} \hat{Z} \oplus N_{[w]}^{*}(\hat{Z} \subset W)$.

For part (ii), we choose coordinates $x_{0}, x_{1}, \ldots, x_{n}$ on $W$ and dual coordinates $y^{0}, y^{1}, \ldots, y^{n}$ on $W^{*}$ such that in the induced coordinates on $V$ the hyperplane $H$ has the equation $x_{0}-y^{n}=0$. Now restrict to the affine piece $x_{0}=y^{n}=1$ on both $H$ and $\mathbb{P}(W) \times \mathbb{P}\left(W^{*}\right)$. We see explicitely, that the projection map $H \rightarrow \mathbb{P}^{2 n-1}$,

$$
\left[1, x_{1}, \ldots, x_{n}, y^{0}, \ldots, y^{n-1}, 1\right] \mapsto\left[y^{1}, \ldots, y^{n-1}, y^{0}-x_{n}, x_{1}, \ldots, x_{n-1}, 1\right]
$$


agrees with the map $\varphi$ from $[6, \S 4]$.

To find singularities of $X$ on $X \cap \mathbb{P}(W)$ as in part (iii) note that $X \subset \mathbb{P}\left(W \oplus W^{*}\right)$ is invariant under the following action of $\mathbb{C}^{*}$ :

$$
t \cdot[w, \alpha]:=\left[t w, t^{-1} \alpha\right] .
$$

In particular points of $X \cap \mathbb{P}(W)$ are fixed points of the action. So let $[w, 0] \in X$ and then $T_{[w, 0]} X$ decomposes into the eigenspaces of the action:

$$
T_{[w, 0]} X=T_{[w, 0]}(X \cap \mathbb{P}(W)) \oplus T_{[w, 0]}\left(X \cap F_{w}\right)
$$

where $F_{w}$ is the fibre of the projection: $\rho:\left(\mathbb{P}\left(W \oplus W^{*}\right) \backslash \mathbb{P}\left(W^{*}\right)\right) \rightarrow \mathbb{P}(W), F_{w}:=$ $\rho^{-1}([w])$. Clearly the image of $X$ under the projection $\rho$ is $Z$, so the dimension of a general fibre of $\left.\rho\right|_{X}: X \rightarrow Z$ is equal to $\operatorname{dim} X-\operatorname{dim} Z=\operatorname{dim} \mathbb{P}(W)-\operatorname{dim} Z=$ $\operatorname{codim}_{\mathbb{P} W} Z$. Therefore, since the dimension of the fibre can only grow at special points, we have:

$$
\operatorname{dim} T_{[w, 0]}\left(X \cap F_{w}\right) \geq \operatorname{dim}\left(X \cap F_{w}\right) \geq \operatorname{codim}_{\mathbb{P}(W)} Z
$$

Also $\mathrm{d}_{[w, 0]}\left(\left.\rho\right|_{X}\right): T_{[w, 0]} X \rightarrow T_{[w]} Z$ maps $T_{[w, 0]}\left(X \cap F_{w}\right)$ to 0 and $T_{[w, 0]}(X \cap \mathbb{P}(W))$ onto $T_{[w]} Z$. Therefore:

$$
\operatorname{dim} T_{[w, 0]}(X \cap \mathbb{P}(W)) \geq \operatorname{dim} T_{[w]} Z \geq \operatorname{dim} Z .
$$

Now assume $[w, 0]$ is a smooth point of $X$. Then adding (3.3) and (3.4) we get:

$$
\begin{aligned}
& \operatorname{dim} X=\operatorname{dim} T_{[w, 0]} X= \\
& \text { by } \stackrel{(3.2)}{=} \operatorname{dim} T_{[w, 0]}\left(X \cap F_{w}\right)+\operatorname{dim} T_{[w, 0]}(X \cap \mathbb{P}(W)) \geq \\
& \geq \operatorname{codim}_{\mathbb{P}(W)} Z+\operatorname{dim} Z=\operatorname{dim} \mathbb{P}(W) .
\end{aligned}
$$

By (i) the very left side is equal to the very right side, so in (3.3) and (3.4) all the inequalities are in fact equalities. In particular $\operatorname{dim} T_{[w]} Z=\operatorname{dim} Z$, so $[w]$ is a smooth point of $Z$.

Conversely, assume $[w]$ is a smooth point of $Z$, then the tangent space

$$
T_{[w, 0]} X=T_{[w]} Z \oplus N_{[w]}^{*}(Z \subset \mathbb{P}(W)),
$$

therefore clearly $[w, 0]$ is a smooth point of $X$.

Exactly the same argument shows that $X$ is singular at a point $[0, \alpha] \in X \cap \mathbb{P}\left(W^{*}\right)$ if and only if $Z^{*}$ is singular at $[\alpha]$.

For the last part of (iii) it is enough to note that $p$ is a locally trivial $\mathbb{C}^{*}$-bundle when restricted to $\mathbb{P}\left(W \oplus W^{*}\right) \backslash\left(\mathbb{P}(W) \cup \mathbb{P}\left(W^{*}\right)\right)$.

Corollary 3.5. Given a Legendrian subvariety $\tilde{Z} \subset \mathbb{P}^{2 n-1}$ we can take $Z^{\#}:=$ $\phi_{H_{0}, p_{0}}^{-1}(\tilde{Z})$ to construct a Legendrian subvariety in $\mathbb{P}\left(T^{*} \mathbb{P}^{n}\right)$. Such a variety must be a conormal variety to some variety $Z \subset \mathbb{P}^{n}$. Let $X \subset \mathbb{P}^{2 n+1}$ be the Legendrian variety constructed above. By theorem 3.1 (ii), a component of a hyperplane section of $X$ can be projected onto $\tilde{Z}$.

Unfortunately, in the setup of the theorem almost always $X$ is singular. 
Example 3.6. If $Z$ is a Kummer quartic surface in $\mathbb{P}^{3}$ then $X$ is a 3-fold with 32 isolated singular points. Therefore by theorem 1.1 a general hyperplane section of $X$ is smooth and admits a Legendrian embedding. By theorem 3.1 the example of Landsberg and Manivel is a special case of this hyperplane section. Even though the condition $p_{0} \in H_{0}$ is a closed condition, it intersects the generality conditions of theorem 1.1 and therefore this hyperplane section consist of a unique smooth component that is projected isomorphically onto $\tilde{Z}$.

Example 3.7. Similarly, if $Z$ is a curve in $\mathbb{P}^{2}$ satisfying generality conditions of Bryant [1, thm G], then $X$ is a surface with only isolated singularities and its hyperplane section projects isomorphically onto Bryant Legendrian curve.

\section{Acknowledgements}

The article is a part of the research project N20103331/2715 funded by Polish financial means for science in years 2006-2008.

This article was written while the author enjoyed the hospitality of Mark Gross at University of California, San Diego and Joseph Landsberg at Texas A\&M University. The author is grateful for their invitation, financial support and for creating a stimulating atmosphere. He acknowledges an inspiration by works of Jarosław Wiśniewski, many discussions with Joseph Landsberg and many precious remarks by Joanna Jaszuńska, Grzegorz Kapustka, Adrian Langer and Andrzej Weber.

\section{References}

[1] R. L. Bryant, Conformal and minimal immersions of compact surfaces into the 4-sphere, J. Differential Geom. 17 (1982), no. 3, 455-473.

[2] J. Buczyński, Legendrian subvarieties of projective space, Geom. Dedicata 118 (2006) 87-103.

[3] — Some quasihomogeneous Legendrian varieties (2007). ArXiv: math.AG/0701750 to appear in Advances in Geometry.

[4] — Toric Legendrian subvarieties, Transform. Groups 12 (2007), no. 4, 631-646.

[5] R. Hartshorne, Algebraic geometry, Springer-Verlag, New York (1977), ISBN 0-387-90244-9. Graduate Texts in Mathematics, No. 52.

[6] J. M. Landsberg and L. Manivel, Legendrian varieties, Asian J. Math. 11 (2007), no. 3, 341-359.

[7] S. Mukai, Curves, K3 surfaces and Fano 3-folds of genus $\leq 10$, in Algebraic geometry and commutative algebra, Vol. I, 357-377, Kinokuniya, Tokyo (1988).

[8] - Simple Lie algebra and Legendre variety (1998). Http://www.math.nagoya-u.ac.jp / mukai/.

E-mail address: jabu@mimuw.edu.pl

Faculty of Mathematics, Informatics and Mechanics, University of Warsaw, Banacha 2, 02-097 Warszawa, Poland 